Published in final edited form as:

Clin Cancer Res. 2020 January 15; 26(2): 397-407. doi:10.1158/1078-0432.CCR-19-2533.

\title{
Clinical utility of plasma cell-free DNA in adult patients with newly diagnosed glioblastoma - a pilot prospective study
}

\author{
Stephen J. Bagley ${ }^{1,2,5}$, S. Ali Nabavizadeh ${ }^{3}$, Jazmine J. Mays ${ }^{1}$, Jacob E. Till ${ }^{1}$, Jeffrey B. \\ Ware $^{3}$, Scott Levy ${ }^{4}$, Whitney Sarchiapone ${ }^{4}$, Jasmin Hussain ${ }^{4}$, Timothy Prior ${ }^{4}$, Samantha \\ Guiry $^{3}$, Theresa Christensen ${ }^{1}$, Stephanie S. Yee ${ }^{1}$, MacLean P. Nasrallah ${ }^{5,6}$, Jennifer J.D. \\ Morrissette $^{6,7}$, Zev A. Binder ${ }^{4,5}$, Donald M. O’Rourke ${ }^{4,5}$, Andrew J. Cucchiara ${ }^{8}$, Steven \\ Brem $^{4,5}$, Arati S. Desai ${ }^{1,2,5}$, Erica L. Carpenter ${ }^{1,2,5}$ \\ 1.Division of Hematology/Oncology, Perelman School of Medicine at the University of \\ Pennsylvania, Philadelphia, PA 19104 \\ 2.Abramson Cancer Center, University of Pennsylvania, Philadelphia, PA 19104 \\ 3.Department of Radiology, Perelman School of Medicine at the University of Pennsylvania, \\ Philadelphia, PA 19104 \\ 4.Department of Neurosurgery, Perelman School of Medicine at the University of Pennsylvania, \\ Philadelphia, PA 19104 \\ 5.Glioblastoma Translational Center of Excellence, Abramson Cancer Center, University of \\ Pennsylvania, Philadelphia, PA 19104 \\ 6. Department of Pathology and Laboratory Medicine, Perelman School of Medicine at the \\ University of Pennsylvania, Philadelphia, PA 19104 \\ 7.Penn Center for Personalized Diagnostics, University of Pennsylvania, Philadelphia, PA 19104 \\ 8. Department of Biostatistics, Epidemiology, and Informatics, Perelman School of Medicine at the \\ University of Pennsylvania, Philadelphia, PA 19104
}

\section{Abstract}

Purpose-The clinical utility of plasma cell-free DNA (cfDNA) has not been assessed prospectively in patients with glioblastoma (GBM). We aimed to determine the prognostic impact

Corresponding author: Stephen J. Bagley, MD, MSCE, $10^{\text {th }}$ Floor Perelman Center South Pavilion, 3400 Civic Center Blvd, Philadelphia, PA 19104, 215-614-1858 (office), 215-662-2432 (fax), Sbagley @ pennmedicine.upenn.edu.

Author Contributions

Conception and design: S.J. Bagley, E.L. Carpenter

Development of methodology: S.J. Bagley, S.A. Nabavizadeh, J.J. Mays, J.E. Till, Z.A. Binder, E.L. Carpenter

Acquisition of data: S.J. Bagley, S.A. Nabavizadeh, J. J. Mays, J.E. Till, J.B. Ware, S. Guiry, S. Levy, W. Sarchiapone, J. Hussain, T. Christensen, S. Brem, D. M. O'Rourke, A.S. Desai, M.P. Nasrallah, J.J.D. Morrissette

Analysis and interpretation of data (e.g., statistical analysis, biostatistics, computational analysis): S.J. Bagley, J.J. Mays, J.E.

Till, A.J. Cucciara, J.I. Odegaard

Writing, review, and/or revision of the manuscript: S.J. Bagley, S.A. Nabavizadeh, J.J. Mays, J. E. Till, S. Brem, Z.A. Binder, D.M. O'Rourke, A.S. Desai, M.P. Nasrallah, A.J. Cucciara, and E.L. Carpenter

Administrative, technical, or material support (i.e., reporting or organizing data, constructing databases): S.J. Bagley, J.J.

Mays, S. Levy, W. Sarchiapone, T. Prior, S.S. Yee

Conflicts of Interest: The authors declare no potential conflicts of interest. 
of plasma cfDNA in GBM, as well as its role as a surrogate of tumor burden and substrate for next-generation sequencing (NGS).

Experimental Design-We conducted a prospective cohort study of 42 patients with newly diagnosed GBM. Plasma cfDNA was quantified at baseline prior to initial tumor resection and longitudinally during chemoradiotherapy. Plasma cfDNA was assessed for its association with progression-free survival (PFS) and overall survival (OS), correlated with radiographic tumor burden, and subjected to a targeted NGS panel.

Results-Prior to initial surgery, GBM patients had higher plasma cfDNA concentration than age-matched healthy controls (mean 13.4 vs. $6.7 \mathrm{ng} / \mathrm{mL}, \mathrm{p}<0.001$ ). Plasma cfDNA concentration was correlated with radiographic tumor burden on patients' first post-radiation magnetic resonance imaging scan $(\rho=0.77, p=0.003)$ and tended to rise prior to or concurrently with radiographic tumor progression. Preoperative plasma cfDNA concentration above the mean $(>13.4 \mathrm{ng} / \mathrm{mL})$ was associated with inferior PFS (median 4.9 vs. 9.5 months, $\mathrm{p}=0.038$ ). Detection of $\geq 1$ somatic mutation in plasma cfDNA occurred in $55 \%$ of patients and was associated with non-statistically significant decreases in PFS (median 6.0 vs. 8.7 months, $\mathrm{p}=0.093$ ) and OS (median 5.5 vs. 9.2 months, $\mathrm{p}=0.053)$.

Conclusions-Plasma cfDNA may be an effective prognostic tool and surrogate of tumor burden in newly diagnosed GBM. Detection of somatic alterations in plasma is feasible when samples are obtained prior to initial surgical resection.

\section{Keywords}

Cell-Free Nucleic Acids; Plasma; Glioblastoma; DNA; Liquid Biopsy

\section{Introduction}

Liquid biopsy, particularly the analysis of plasma cell-free DNA (cfDNA), has emerged as a minimally invasive tool for molecular profiling and tumor monitoring in clinical oncology (1). In patients with cancer, circulating tumor DNA (ctDNA) shed by cancer cells constitutes only a portion of the overall cfDNA (2), which is short fragment (usually 130-180 base pairs), double- stranded DNA that is normally present in the bloodstream and can fluctuate physiologically in response to exercise, cellular injury, or inflammation (3). Even when the fraction of ctDNA in the larger cfDNA pool is low, this material can be used as a substrate for tumor molecular profiling in cancer patients $(4,5)$, including mutation detection by nextgeneration sequencing (NGS) to identify sensitivity and resistance to targeted therapies (69). In addition, due to their short half-life (minutes to hours) in the plasma, both ctDNA $(10,11)$ and cfDNA $(12,13)$ have been studied as real-time biomarkers of tumor burden, providing a minimally invasive means of disease monitoring in response to antineoplastic therapy. Prior research has also demonstrated a role for plasma cfDNA and ctDNA levels, either at a single time point or longitudinally, as prognostic and/or predictive of treatment response in numerous tumor types (14-16).

As liquid biopsy using ctDNA, extracellular vesicles, and other circulating tumor material (17) has been adopted rapidly across multiple solid tumors, use of this technology has been limited in glioblastoma (GBM), the most common primary malignant brain tumor in adults 
(18). Although the use of plasma ctDNA in GBM is particularly appealing, due to both its marked molecular heterogeneity (19) and the highly invasive nature of brain surgery, initial enthusiasm for the use of plasma ctDNA in GBM was muted by a study demonstrating low yield of plasma ctDNA in patients with glioma compared to other solid tumors (20). However, this included only 27 patients with glioma, nearly half of which were grade II or III. Plasma cfDNA yield in these patients may have been limited by the lower rates of cell proliferation, lack of necrosis, and greater blood-brain barrier integrity in low grade gliomas compared to GBM (21). This hypothesis is supported by several studies, including one that detected isocitrate dehydrogenase-1 (IDH1) p.R132H mutations by plasma droplet digital PCR (ddPCR) in 15 out of 25 patients (60\%) with IDH1-mutated gliomas (22). Notably, larger tumor volumes and higher histologic grade were associated with increased plasma cfDNA concentration and more frequent detection of the p.R132H mutation. In a separate study, the histone 3 p.K27M (H3K27M) mutation was successfully detected by ddPCR in the plasma of 42 out of 48 pediatric patients ( $88 \%$ ) with high-grade diffuse midline gliomas (23). Other studies using a targeted NGS panel on ctDNA have demonstrated detection of at least one somatic alteration in the plasma of between $27-55 \%$ of adult patients with GBM $(24,25)$. However, these studies lacked clinical information for the vast majority of patients, most notably the timing of plasma collection in relation to the patients' therapies, the association between cfDNA/ctDNA and clinical outcomes, and the results of concurrent tumor NGS.

At present, there remains a paucity of data regarding the detection and potential clinical utility of plasma cfDNA when collected (a) exclusively in GBM (grade IV) patients, and (b) at times of high tumor burden when cfDNA yield may be highest, such as prior to surgical resection. The primary objective of this prospective pilot study was to assess the feasibility of serial, longitudinal plasma cfDNA collection and quantification in patients with newly diagnosed GBM from the time of initial radiographic suspicion of high-grade glioma (i.e, prior to surgery) through the time of disease progression on standard radiation and temozolomide. In addition, we sought preliminary insight into the clinical utility of plasma cfDNA in GBM, including for the detection of somatic tumor mutations, as a prognostic marker, and as a surrogate of tumor burden to aid in discrimination between true tumor progression and pseudoprogression (26). We hypothesized that high plasma cfDNA concentration portends worse prognosis in newly diagnosed GBM, that plasma cfDNA concentration is correlated with GBM radiographic tumor burden, and that GBM tumor somatic mutations can be readily detected in plasma specimens collected prior to initial surgical resection.

\section{Materials and Methods}

\section{Study Design and Patient Population}

Within the context of a larger institutional plasma cfDNA banking protocol in patients with tumors of the central nervous system (CNS), we performed a prospective cohort study of adult patients with newly diagnosed GBM. The study was conducted in accordance with the Declaration of Helsinki, and all applicable laws. Potential subjects were screened from the inpatient and outpatient Neurosurgery practices at the University of Pennsylvania on the 
basis of having a magnetic resonance imaging (MRI) scan suspicious for high-grade glioma and a plan for a diagnostic and/or therapeutic surgical procedure. All subjects meeting these criteria and providing informed consent had pre-operative whole blood samples collected in the operating room prior to skull opening. In addition, baseline demographic and clinical/ laboratory variables were collected, including age, sex, IDH1/2 mutational status, O6methylguanine-methyltransferase (MGMT) promoter methylation, Karnofsky performance status (KPS), extent of surgical resection, and serum creatinine for calculation of glomerular filtration rate (GFR). Subjects remained on the longitudinal component of the study after surgery if the following criteria were met: (a) histopathology confirmed a diagnosis of GBM, (b) the subject was planned for standard radiation with concurrent and adjuvant temozolomide (27), and (c) the subject consented to continued serial blood draws during the course of their adjuvant treatment. For subjects continuing on the longitudinal arm, whole blood was collected for cfDNA analysis between 24-48 hours postoperatively, and then on days of all future MRI scans, including MRIs obtained for radiation planning, 1 month after completion of radiation, and approximately every 2 months thereafter (or sooner if clinical disease progression was suspected). In order to obtain a control population of healthy patients, we also drew whole blood for plasma cfDNA extraction and quantification from 42 healthy donor patients who were approached for consent in a routine screening upper/lower endoscopy clinic at our institution. Inclusion criteria for the healthy donor cohort were age 18 or older, non-pregnant, no known diagnoses of cancer, viral hepatitis, HIV infection, or systemic inflammatory conditions, and no recent surgical procedures or trauma. All subjects provided written informed consent approved by the Institutional Review Board of the University of Pennsylvania.

\section{Specimen Collection and Plasma Isolation}

Whole blood samples were collected in Streck cfDNA blood collection tubes and processed to plasma and frozen within 96 hours. Whole blood was centrifuged at $1600 \mathrm{xg}$ for $10 \mathrm{~min}$. The plasma supernatant was isolated and centrifuged twice at $4122 \mathrm{x} \mathrm{g}$ for $15 \mathrm{~min}$ (swinging bucket, break-off), aliquoted, and frozen at $-80 \mathrm{C}$ for future use.

\section{cfDNA extraction and quantification}

cfDNA was extracted from $1 \mathrm{~mL}$ of thawed plasma using the QIAamp Circulating Nucleic Acid Kit according to the manufacturer's protocol (QIAGEN \#55114) with the following deviation: buffer-lysate solution was incubated at $60 \mathrm{C}$ for 1 hour instead of 30 minutes and cfDNA was eluted twice with 30uL of Buffer AVE each. Extracted cfDNA was stored at 4C for short-term use or long-term at -20C. cfDNA concentration $(\mathrm{ng} / \mathrm{mL})$ was quantified by SYBR Green-based qPCR for a 115 bp amplicon of the human ALU repeat element (28) with forward primer 5'- CCT GAG GTC AGG AGT TCG AG -3' and reverse primer 5'CCC GAG TAG CTG GGA TTA CA -3' (Integrated DNA Technologies). In brief, DNA standard (Promega \#PAG3041) curve dilutions and samples were diluted 1:10 with nucleasefree water and qPCR carried out in quadruplicate using Power SYBR Green PCR Master Mix (Applied Biosystems \#4367659) according to the manufacturer's instructions on a ViiA 7 Real-Time PCR System (Applied Biosystems). Results were analyzed using QuantStudio Real-Time PCR Software (Applied Biosystems). 


\section{Histopathologic evaluation}

Tumor specimens from initial surgical resection in each of the subjects were assessed by a board-certified neuropathologist (M.P.N.). The following factors related to tumor proliferation, necrosis, and/or abnormal vasculature were quantified in each specimen: the overall tumor Ki-67 proliferation index (semi-quantitatively estimated based on inspection of a stained section from one block, excluding positive cells histologically compatible with inflammatory cells), microvascular proliferation (graded 0-6; none to extensive), pseudopalisading necrosis (graded 0-6; none to extensive), and the estimated percentage of the specimen comprised of geographic necrosis. Ki-67 immunohistochemistry was performed on the Bond 111 autostainer with the Dako mouse monoclonal antibody (IR62661, clone MIB-1). Unstained slides were pretreated with heat retrieval, epitope retrieval 1, in citrate buffer $\mathrm{pH} 6.0$ (Leica Microsystems) for 20 minutes.

\section{Next-generation sequencing}

All subjects had targeted NGS of their treatment-naïve tumor tissue by the University of Pennsylvania's Clinical Laboratory Improvement Amendments (CLIA)-certified Center for Personalized Diagnostics laboratory using a 152-gene panel (Comprehensive Solid Tumor HaloPlex ${ }^{\mathrm{HS}}$, version 2.0; Agilent Technology, Inc; Supplementary Table S1), as previously described $(4,7)$. Mutations were classified as disease-associated (either pathogenic or probably disease-associated), variant of uncertain significance, likely benign, or benign based on review of the literature and publicly available databases. Only disease-associated mutations are included in this analysis. Tissue sequencing also included an institutional 55gene fusion transcript panel (Archer Dx, Boulder, CO), which uses anchored multiplex priming and NGS (Illumina HiSeq) to identify aberrant transcripts from RNA (Supplementary Table S2). In a subset of 20 patients who provided additional consent for third-party sequencing of plasma cfDNA, plasma samples obtained prior to initial surgical resection were sequenced by Guardant Health [Guardant360, as previously described $(4,7)$ ] to compare mutations detected in plasma with those detected in tissue. The current Guardant360 panel used in this study (Supplementary Table S3) requires minimum input quantity of $5 \mathrm{ng}$ of cfDNA and assesses for single-nucleotide variants (SNV) and insertiondeletions (indel) in 74 genes, and fusion alterations and copy-number amplifications in select genes. The exons of these 74 genes were captured using biotinylated custom bait oligonucleotides (Agilent, Santa Clara, CA), resulting in a capture footprint of 148,000 base pairs (78 kb). Samples were paired-end sequenced on an Illumina HiSeq 2500 (San Diego, $\mathrm{CA}$ ), followed by algorithmic reconstruction of the digitized sequencing signals. Illumina sequencing reads were mapped to the hg19/GRCh37 human reference sequence, and genomic alterations in cfDNA were identified from Illumina sequencing data by proprietary bioinformatic algorithms. These algorithms quantify the absolute number of unique DNA fragments at a given nucleotide position, thereby enabling ctDNA to be quantitatively measured as a fraction of total cfDNA. The Guardant 360 cfDNA test has demonstrated extensive analytical and clinical validity and utility $(7,29,30)$. Overlap in gene coverage between the tissue and plasma NGS panels is displayed in Supplementary Tables S1-S3. The Guardant360 plasma panel has greater depth of sequencing (average depth of $\sim 15,000 \mathrm{x}$ ), compared to $\sim 2,500 \mathrm{x}$ for our tissue panel. 


\section{Radiographic Assessment}

Brain MRI was performed on a 3-T MR imaging unit (Magnetom TrioTim; Siemens, Erlangen, Germany) by using a 12-channel phased array head coil. Routine sequences were obtained including volumetric axial T1-weighted 3D MPRAGE $(\mathrm{TR} / \mathrm{TE} / \mathrm{TI}=1760 / 3.1 / 950$ ms, $192 \times 256$ matrix size, 1 -mm section thickness) before and after contrast and post contrast axial FLAIR (TR/TE/TI = 9420/141/2500 ms, 3-mm section thickness). Tumor volumes were used to report tumor burden in light of recent data suggesting that threedimensional volumetry may provide more accurate assessment of tumor burden and response in GBM than standard two-dimensional Response Assessment in Neuro-Oncology (RANO) measurements (31).

Structural MRI data were analyzed at each individual timepoint for every subject by two board-certified neuroradiologists (S.A.N. and J.B.W.) who were blinded to cfDNA values. For the preoperative timepoint, both T2 FLAIR and T1 post-contrast MRI images were evaluated visually for regions of abnormal FLAIR signal intensity and contrast enhancement. These sequences were used for segmentation of nonenhancing and enhancing tumor, respectively, in a semi-automated fashion using ITK-SNAP (32). The post-operative time points were analyzed similarly, except that areas of hemorrhage identified on precontrast sequences were also segmented and subtracted from the final tumor segmentation masks. The numerical volumes of the resulting enhancing tumor and edema segmentations were then compiled and analyzed.

In a subset of patients, dynamic contrast-enhanced (DCE) imaging was performed using a gradient echo T1-weighted sequence (TR/TE 4.8/1.47 ms, flip angle $23^{\circ}$, voxel size $0.86 \times$ $0.86 \times 3 \mathrm{~mm}$, temporal resolution $7 \mathrm{~s}$ ) following a bolus injection of gadolinium-containing contrast agent with a dose of $0.1 \mathrm{mmol} / \mathrm{kg}$. The modified Tofts model was applied to DCE data using the MATLAB software package to generate the volume transfer constant $\left(\mathrm{K}^{\text {trans }}\right)$ (33).

\section{Statistical Analysis}

Mean baseline cfDNA concentration was compared between healthy donors and patients with GBM using an unpaired t-test. Comparisons between subjects with high vs. low baseline plasma cfDNA were performed with Fisher's exact tests or chi-square tests for categorical variables, as appropriate, and Mann-Whitney $U$ tests for continuous variables. The correlation between plasma cfDNA concentration and volumetric tumor burden by MRI was assessed at multiple time points, including baseline (pre-surgery), radiation simulation, and one month post-radiation. Spearman's correlation coefficient was used given that the distribution of tumor volumes was highly skewed. Beyond radiographic tumor burden, other demographic, clinical, radiologic, and histopathologic parameters were evaluated for their association with baseline plasma cfDNA concentration using simple linear regression. Variables associated with cfDNA concentration in univariate analysis with p-value $\leq 0.15$ were entered into a multiple linear regression model. We tested for a difference in plasma cfDNA concentration between patients with and without somatic alterations detected in plasma using an unpaired t test. 
The Kaplan-Meier (KM) method was used to estimate median progression-free survival (PFS) and overall survival (OS). The reverse KM method was used to calculate median follow-up time. A log-rank test was used to test for crude differences in survival between subjects with baseline plasma cfDNA concentration above vs. below the mean value for the cohort ("high cfDNA" vs. "low cfDNA"), as well as between subjects with vs. without somatic variants detected in the plasma. PFS time was defined as the number of months from the date of initial surgery/biopsy for GBM until the date of tumor progression or death, whichever occurred first (or date of last follow up if progression or death had not yet occurred). The date of tumor progression was determined retrospectively at the time of the data cut-off for this analysis and was defined using all available data from the electronic health record, including clinician notes, radiology reports from serial MRI scans, and pathology reports from repeat biopsies/surgeries when available. Using these data sources, tumor progression was declared if there was radiographic progression of disease according to RANO criteria, AND at least one of the following events occurred as a result of the MRI scan that met criteria for progression: (a) the subject underwent repeat surgical resection with histopathology that demonstrated viable, recurrent glioblastoma, (b) there was consensus agreement in multidisciplinary tumor board conference that the MRI findings represented tumor progression, or (c) there was clinical decline necessitating a change in treatment. These criteria were implemented to account for the possibility of pseudoprogression, which is a common phenomenon in GBM patients treated with radiation (26). OS was defined as the number of months from the date of initial surgery/biopsy for GBM until the date of death (or date of last follow up if death had not yet occurred). Cox proportional hazards $(\mathrm{PH})$ regression was used to adjust for relevant prognostic variables including age (< 65 vs. $\geq 65$ ), IDH1/2 mutational status (positive vs. negative), $M G M T$ promoter methylation status (methylated vs. unmethylated), extent of surgical resection (gross/near total resection vs. subtotal resection or biopsy only), and KPS ( $\leq 60$ vs. 270 ).

Within each individual subject with at least 4 time points available for MRI and cfDNA collection (pre-operative, post-operative, radiation simulation, and 1-month post-radiation; one subject excepted due to early disease progression by the third time point), line graphs were generated to display the relationship between changes in radiographic tumor burden and plasma cfDNA concentration over time. A p-value $<0.05$ was considered statistically significant. All tests were 2-tailed. Stata Statistical Software (Version 14) was used for statistical analyses (StataCorp 2015). There was no missing data in this study, with the exceptions of DCE MRI ( $\left.k^{\text {trans }}\right)$, which was only available for 15 subjects, and plasma NGS, which was performed in only 20 subjects.

\section{Results}

\section{Plasma cfDNA concentration is elevated and correlated with radiographic tumor burden in patients with newly diagnosed GBM}

The distribution of baseline (pre-operative) plasma cfDNA concentrations in subjects with newly diagnosed GBM and comparison to age-matched (+/ -3 years) healthy controls is displayed in Fig. 1. Median age was 65 (range 20-81) in the GBM cohort and 62 (range 2282 ) in the healthy cohort. GBM patients had higher plasma cfDNA concentration vs. healthy 
donors (mean $13.4 \mathrm{ng} / \mathrm{mL}$ vs. $6.7 \mathrm{ng} / \mathrm{mL}, \mathrm{p}<0.0001$ ). Demographic and clinicopathologic characteristics of the GBM cohort are displayed in Table 1, including comparison of subjects with baseline plasma cfDNA concentration above vs. below the mean value. Other than a slightly lower proportion of subjects with EGFR mutations and higher proportion of subjects with $M G M T$ promoter methylation than expected, the overall cohort is representative of a typical GBM population (18). Twenty-six subjects (62\%) received 60 Gy radiation and 16 $(38 \%)$ received 40 Gy. Thirty-four subjects $(81 \%)$ received temozolomide concurrent with radiation and as maintenance therapy following radiation, and 10 subjects (24\%) used tumor-treating fields during adjuvant therapy. There were no statistically significant differences in baseline characteristics between the subjects with high vs. low baseline plasma cfDNA concentration (Table 1).

At the time of initial radiographic suspicion of GBM (i.e., prior to initial surgical resection) and at the time of radiation simulation, there was minimal to no association between plasma cfDNA concentration and total radiographic tumor burden, defined as the sum of the volumes of $\mathrm{T} 1$ post-contrast enhancing tumor and T2/FLAIR signal abnormality (presurgery, $\rho=0.24, p=0.13$; radiation simulation, $\rho=-0.09, p=0.79$ ). In addition, no correlation was detected at these time points when the volumes of contrast-enhancing tumor or T2/ FLAIR signal abnormality, respectively, were considered in isolation. However, in the 12 subjects who had volumetric measurements performed on the 1-month post-radiation MRI by the time of the data cut-off for this analysis, there was a significant correlation between plasma cfDNA concentration drawn at that time and total radiographic tumor burden $(\rho=0.77, p=0.003)$ and between plasma cfDNA concentration and contrast-enhancing radiographic tumor burden $(\rho=0.62, p=0.03)$ (Supplementary Fig. S1).

The association of other baseline characteristics with pre-operative plasma cfDNA concentration was assessed using simple linear regression. In univariate analysis, only age (coefficient 0.27, $\mathrm{p}=0.03$ ), $M G M T$ promoter methylation (coefficient $-6.1, \mathrm{p}=0.07$ ), and GFR $(-0.11, \mathrm{p}=0.05)$ were associated with plasma cfDNA concentration. However, none of these variables remained associated with cfDNA when combined into a multiple linear regression model. In addition, no histopathologic parameters (Ki-67 proliferation index, $\mathrm{p}=0.16$; microvascular proliferation, $\mathrm{p}=0.30$; geographic necrosis, $\mathrm{p}=0.28$; pseudopalisading necrosis, $\mathrm{p}=0.71$ ) or DCE-MR imaging measurements $\left(\mathrm{k}^{\text {trans }}, \mathrm{p}=0.50\right)$ were associated with plasma cfDNA concentration.

\section{High baseline plasma cfDNA is independently associated with inferior outcomes}

To examine the prognostic role of baseline plasma cfDNA concentration in newly diagnosed GBM, we divided the GBM cohort into two groups (high cfDNA vs. low cfDNA) based on whether a subject's plasma cfDNA concentration prior to initial surgical resection was above or below the mean $(13.4 \mathrm{ng} / \mathrm{mL})$. Of note, the mean cfDNA value was used to dichotomize the GBM population (rather than median) based on the distribution of baseline cfDNA values in this cohort compared to healthy controls (Fig. 1). The mean value of cfDNA concentration in the GBM cohort was $13.4 \mathrm{ng} / \mathrm{mL}$, which corresponded almost exactly to the highest value from a healthy donor $(13.5 \mathrm{ng} / \mathrm{mL})$, with the exception of one extreme outlier in the healthy control population. Thus, compared to the median value $(10.5 \mathrm{ng} / \mathrm{mL})$, we 
observed that the mean value of cfDNA in the GBM population seemed to better capture the cutoff point for identifying GBM patients with cfDNA concentrations that are truly elevated, i.e., beyond what would be expected in a healthy population.

Median follow-up time for PFS by the reverse KM method was 11.3 months (95\% CI 9.112.1 months). Median PFS was 7.4 months (95\% CI 5.5 - 10.9 months). Subjects with GBM and high baseline cfDNA according to our cut point $(>13.4 \mathrm{ng} / \mathrm{mL}$ ) had worse PFS (Fig. 2A), with a median PFS of 4.9 months (95\% CI 1.5 - 7.8 months) compared to 9.5 months (95\% CI $6.4-12.8$ months) in subjects with low cfDNA (log-rank p=0.038). After adjusting for known prognostic factors, including age, IDH mutational status, $M G M T$ promoter methylation, KPS, and extent of surgical resection, high baseline plasma cfDNA remained independently associated with inferior PFS (HR 2.48, 95\% CI $1.02-6.05, \mathrm{p}=0.046$ ). Median OS was 14.8 months (95\% CI 8.5 - NR). Although only less than half of the subjects in our GBM cohort had died at the time of the data cut-off, there was also a non-statistically significant trend toward inferior OS in subjects with high baseline plasma cfDNA (median OS 8.9 vs. 14.8 months, log-rank p=0.12; Fig. 2B). The final multivariate Cox PH model for OS, adjusted for the same prognostic factors listed above, yielded a HR of 1.82 for high cfDNA ( $95 \%$ CI $0.61-5.42, \mathrm{p}=0.28$ ).

\section{Targeted NGS of plasma cfDNA}

Among the 20 patients who had plasma that was collected preoperatively and sequenced by targeted NGS, 11 patients (55\%) had at least 1 somatic mutation detected (Supplementary Table S4). The mean allele fraction (AF) of somatic alterations detected in the plasma was $0.84 \%$. There was no difference in the mean cfDNA concentration between patients with vs. without a somatic variant detected in the plasma (mean concentration $18.2 \mathrm{vs.} 13.0 \mathrm{ng} / \mathrm{mL}$; $\mathrm{p}=0.39$ ). Matched tissue collected from the initial surgical resection was also subjected to targeted NGS. Fifteen patients (75\%) had $\geq 1$ alteration detected by tissue NGS (Supplementary Table S4). Variants covered by both the plasma and tissue NGS panels are displayed for each subject in Fig. 3. All variants detected in plasma were covered by the tissue NGS panel. All variants detected in tissue were covered by the liquid biopsy panel, with the exception of three mutations in genes for which the liquid biopsy panel has only partial exon coverage ( $N F 1$ R1276Q, NF1 L1253Pfs*9, and PTPN11 G503R). As expected due to the broader coverage of the tumor DNA versus the ctDNA panels, the mean number of variants detected per patient by tumor DNA was 2.1 , compared to 0.5 using ctDNA. There were no mutations detected by both tumor NGS and ctDNA NGS in any patient, although two patients had different variants in the same gene detected by both platforms (TP53 and PDGFRA mutations, respectively; Fig. 3). Detection of a somatic variant in plasma was associated with inferior PFS (Fig. 2C; median 6.0 vs. 8.7 months; $\mathrm{p}=0.093$ ) and OS (Fig. $2 \mathrm{D}$; median 5.5 vs. 9.2 months, $\mathrm{p}=0.053$ ), although these did not reach statistical significance.

\section{Plasma cfDNA dynamics during radiation and chemotherapy}

The potential role of plasma cfDNA for monitoring disease progression was explored in the 12 subjects who had longitudinal blood samples collected starting before initial surgery for GBM and through completion of standard adjuvant radiotherapy. For this analysis, subjects 
were defined as either progressors or nonprogressors depending on whether or not criteria for tumor progression (as outlined in the Methods section) had been met prior to the data cutoff for this analysis. In general, plasma cfDNA concentrations were labile and often increased in the post-operative and radiation planning periods and did not track closely with radiographic tumor burden at these time points (Fig. 4 and Fig. 5). However, for the 6 subjects determined not to have had disease progression (nonprogressors), the plasma cfDNA concentration tended to remain stably low during the course of adjuvant therapy, with only minor fluctuations (Fig. 4). In the 6 subjects who experienced progressive disease prior to the data cutoff (with 2 of these 6 subjects [A048 and A057] having repeat surgery confirming recurrent GBM), all subjects experienced a rise in plasma cfDNA concentration (at least doubling in 5 of 6 subjects) around the time of radiographic evidence of tumor progression (Fig. 5). In all subjects but one, the rise in cfDNA occurred prior to or concurrent with radiographic progression; in the other subject (A057), the rise in cfDNA lagged shortly behind radiographic progression. Clinical details for each subject, including steroid and bevacizumab use, which could potentially affect cfDNA yield, are described in the figure captions (Fig. 4 and Fig. 5).

\section{Discussion}

In this study, we demonstrate that baseline and longitudinal quantification of plasma cfDNA in patients with newly diagnosed GBM is feasible and may have clinical utility. Specifically, we show that high plasma cfDNA concentration prior to initial surgical resection is independently associated with worse outcomes in patients with GBM undergoing standard adjuvant chemoradiotherapy. Our data also suggest a potential role for plasma cfDNA as a noninvasive biomarker of tumor burden in GBM, as we demonstrated (a) a strong correlation between plasma cfDNA concentration and radiographic tumor burden at the time of the first post-radiation MRI in these patients, and (b) a significant rise in plasma cfDNA concentration coinciding with radiographic increase in tumor volume in most patients who had tumor progression. Lastly, we showed that at least one tumor somatic mutation could be detected in the majority of patients with newly diagnosed GBM when collected prior to initial surgical resection.

To our knowledge, this is the first study to show a prognostic role for plasma cfDNA in GBM. Previous studies have shown similar results in other solid tumors. High plasma cfDNA concentration, consisting of both non-tumor-and tumor-derived cfDNA and unrelated to the identification of specific mutations, has been associated with inferior outcomes in pancreas cancer (34), non-small cell lung cancer (NSCLC) (4), colorectal cancer (35), and melanoma (12). Despite the small sample size and relatively low number of PFS/OS events in our cohort to date, high baseline plasma cfDNA concentration predicted worse PFS that was independent of other known prognostic factors in newly diagnosed GBM. The cellular mechanisms driving increased plasma cfDNA in patients with GBM, including the mechanism of tumor-derived ctDNA shedding into the circulation, are not well-characterized. In other tumor types, previously suggested mechanisms include active secretion by tumor cells (36), as well as tumor apoptosis and necrosis (37). We were unable to identify any demographic or clinicopathologic variables that were associated with baseline plasma cfDNA concentration, including tumor size, mutations detected by tissue or 
plasma NGS, or extent of necrosis on histopathology. In addition, MRI assessment of bloodbrain barrier permeability ( $\left.\mathrm{k}^{\text {trans }}\right)$ had no association with cfDNA concentration. This is consistent with a prior animal study, which demonstrated that the integrity of the blood-brain barrier does not affect the release of ctDNA in treatment-naïve GBM (38). Taken together, our data suggest that high baseline plasma cfDNA concentration is reflective of aggressive underlying tumor biology that is not captured by known prognostic factors and portends worse response to standard chemoradiotherapy in GBM. Additional studies are needed to determine the precise mechanism through which elevated cfDNA is associated with worse prognosis in GBM and other solid tumors.

To our knowledge, our study is also the first to compare alterations detected in matched tissue and plasma from patients with newly diagnosed GBM. We showed that plasma cfDNA collected prior to initial surgical resection for GBM can be sequenced with detection of at least one somatic mutation in the majority of patients. The array of mutations detected in our study by plasma NGS is consistent with what would be expected based on prior tissue-based analysis of the somatic genomic landscape of GBM (39). Moreover, mutations were detected in a number of potentially therapeutically relevant genes, including TP53, $E G F R, E R B B 2, P D G F R A$, and NF1. These results question the current paradigm that detection of ctDNA in the plasma of patients with GBM is futile (20). However, none of the mutations detected in plasma were identified in matched tissue samples subjected to NGS. These results may be explained in part by tumor heterogeneity, which has been invoked in multiple other solid tumor studies as a potential cause of tissue-plasma discordance $(4,7,40)$. GBM is characterized by marked molecular heterogeneity, which has been described even on a single-cell level within a single focus of tumor (19). Clinical tissue sequencing of GBM tumors typically relies on isolation of DNA from one small part of a tumor, thus detecting only mutations that are present in that particular sample. The discovery of mutations by plasma NGS that would have gone undetected by tissue sequencing in over half of our patients suggests that combined use of tissue and plasma NGS may provide a more comprehensive assessment of tumor molecular heterogeneity in GBM, which is considered one of the most significant barriers to precision medicine in this disease (41). Importantly, in the absence of NGS results for matched buffy coats, we cannot rule out the possibility that some mutations detected in cfDNA might be reflective of clonal hematopoiesis (CHP), especially those in genes such as $J A K 2$ previously associated with CHP (42). In addition, some of the mutations detected may represent subclonal passenger events that do not carry biologic or clinical relevance. Despite this limitation, the inferior survival of patients in our cohort who had a somatic variant detected in plasma suggests that there is tumor-derived DNA in the circulation of these patients and supports the hypothesis that at least some of the mutations detected in plasma reflect true tumor somatic variants. Larger studies are needed to confirm our results and explore the therapeutic implications of plasma-based molecular profiling in GBM.

In addition to the detection of mutations in plasma that were not present in tumor (i.e., "false positives"), the sensitivity of plasma NGS for detecting mutations identified in matched tissue specimens was $0 \%$. Although this result should be interpreted with caution given the small sample size, the lack of plasma-based detection of known mutations identified in tissue (i.e., "false negatives") suggests that plasma NGS should not be used as a substitute or 
surrogate for tissue NGS in GBM. The most likely explanation for this finding is the low amount of tumor DNA present in the plasma of GBM patients compared to patients with metastatic solid tumors (20). Prior studies have demonstrated higher false negative rates for plasma NGS when variant allele frequency (VAF) is less than $1 \%$, and the mean VAF of plasma alterations detected in our study was $0.84 \%$ (43). One way to improve the sensitivity of liquid biopsy for detecting mutations identified in GBM tumors may be through sampling of cerebrospinal fluid (CSF), as previous studies have demonstrated a high rate of mutation detection in the CSF of patients with CNS tumors (44-46). However, procurement of CSF requires an invasive procedure, such as lumbar puncture or intraoperative sampling from the ventricles, both of which are not performed in the clinical care of the vast majority of patients with GBM. Various approaches currently under investigation may soon lead to improved detection of plasma ctDNA in GBM, resulting in better concordance with tissue sequencing. These include focusing on a specific subset of shorter fragment lengths from cfDNA (47), measurement of plasma cell-free mitochondrial tumor DNA (38), or use of focused ultrasound to transiently open the blood-brain barrier (48).

We also found that a longitudinal increase in plasma cfDNA may serve as a surrogate of tumor progression in patients with GBM. In numerous non-CNS tumors, including NSCLC, breast cancer, colorectal cancer, melanoma, prostate cancer, pancreas cancer, and head and neck cancer, cfDNA (either tumor-derived DNA, expressed as mutant allele fraction, or a mixture of tumor-derived and non-tumor-derived DNA, expressed as raw cfDNA quantification) has been shown to correlate with tumor burden and can be used as a noninvasive and rapid assessment of response to therapy (10-13). Similar results were also recently described using plasma ctDNA for detection of H3K27M mutations and noninvasive tumor surveillance in pediatric patients with diffuse midline glioma (23). In our study, although cfDNA concentration did not correlate closely with radiographic tumor burden immediately pre- and post-operatively in patients with newly diagnosed GBM, there was a strong correlation between radiographic tumor burden and plasma cfDNA concentration at the first post-radiation MRI, an imaging time point that has recently been shown to be a better baseline for evaluating therapeutic efficacy in newly diagnosed GBM (49). The reason for this discrepancy is not clear, although prior work in non-cancer populations has suggested that plasma cfDNA levels fluctuate significantly in the perioperative period due to cellular trauma, inflammation, and effects of anesthetic agents (50). We also found that, in most patients with GBM who experienced disease progression during standard adjuvant chemoradiotherapy, there was a rise in plasma cfDNA concentration concurrently with or prior to radiographic evidence of tumor progression. Conversely, cfDNA tended to remain low and with only minor fluctuations in patients who did not experience disease progression. Although the results of this pilot study must be confirmed in larger studies, our data suggests that plasma cfDNA should be further evaluated as a noninvasive biomarker of tumor burden and disease progression in patients with GBM. Identification of such a biomarker is critically important in GBM due to the invasive nature of repeated brain surgeries, as well as the limited ability of brain MRI to distinguish between true tumor progression and pseudoprogression (26).

Our study has additional limitations not already described. First, the small sample size of this single-center pilot study precludes the ability to draw definitive conclusions about the 
operating characteristics of plasma cfDNA as a biomarker in patients with GBM. A validation cohort is necessary to confirm our findings, and we have continued to enroll patients on this study to validate our results. Nonetheless, our initial findings provide novel proof of principle that plasma cfDNA may have clinical utility in GBM, including as a prognostic marker, a surrogate of tumor burden, and a noninvasive means of molecular profiling. Second, tumor progression in our study was determined in the absence of histologic confirmation of progressive/recurrent tumor in most patients. Instead, serial MRI scans and clinical history (including clinical decline, outcomes of multidisciplinary tumor board discussions, and start of new antineoplastic therapies) were used to determine the date of progression. Although tissue would ideally be obtained in all cases, this is not practical in recurrent GBM due to the invasive nature of brain surgery. Furthermore, our approach for assessing progression, which leveraged the extensive information available through retrospective chart review, allowed for a more comprehensive assessment of disease status than is typically possible when assessing tumor response in real-time. Thus, we suspect that our determination of tumor progression was highly accurate.

In conclusion, our findings suggest that plasma cfDNA may serve as a noninvasive biomarker of tumor burden and prognosis in patients with GBM, as well as a substrate for molecular profiling that may complement tissue sequencing. Larger studies are warranted to confirm the clinical utility of plasma cfDNA for patients with this disease.

\section{Supplementary Material}

Refer to Web version on PubMed Central for supplementary material.

\section{Acknowledgements}

The authors would like to acknowledge the generosity of all patients and their families. We would also like to thank Eileen Maloney-Wilensky and all members of the University of Pennsylvania Neurosurgery Clinical Research Division for their support.

This work was supported by the National Center for Advancing Translational Sciences of the NIH under award number UL1TR001878, the Paul Calabresi Career Development Award for Clinical Oncology NIH K12 grant (K12 CA076931), and the Abramson Cancer Center Glioblastoma Translational Center of Excellence. The plasma cfDNA sequencing in this study was partially funded by Guardant Health (Redwood City, CA).

\section{References}

1. Corcoran RB, Chabner BA. Application of Cell-free DNA Analysis to Cancer Treatment. N Engl J Med 2018;379(18):1754-65 doi 10.1056/NEJMra1706174. [PubMed: 30380390]

2. Diehl F, Schmidt K, Choti MA, Romans K, Goodman S, Li M, et al. Circulating mutant DNA to assess tumor dynamics. Nat Med 2008;14(9):985-90 doi 10.1038/nm.1789. [PubMed: 18670422]

3. Leon SA, Shapiro B, Sklaroff DM, Yaros MJ. Free DNA in the serum of cancer patients and the effect of therapy. Cancer Res 1977;37(3):646-50. [PubMed: 837366]

4. Thompson JC, Yee SS, Troxel AB, Savitch SL, Fan R, Balli D, et al. Detection of Therapeutically Targetable Driver and Resistance Mutations in Lung Cancer Patients by Next-Generation Sequencing of Cell-Free Circulating Tumor DNA. Clinical cancer research : an official journal of the American Association for Cancer Research 2016;22(23):5772-82 doi

10.1158/1078-0432.Ccr-16-1231. [PubMed: 27601595] 
5. Newman AM, Bratman SV, To J, Wynne JF, Eclov NC, Modlin LA, et al. An ultrasensitive method for quantitating circulating tumor DNA with broad patient coverage. Nat Med 2014;20(5):548-54 doi 10.1038/nm.3519. [PubMed: 24705333]

6. Diaz LA Jr., Williams RT, Wu J, Kinde I, Hecht JR, Berlin J, et al. The molecular evolution of acquired resistance to targeted EGFR blockade in colorectal cancers. Nature 2012;486(7404):53740 doi 10.1038/nature11219. [PubMed: 22722843]

7. Aggarwal C, Thompson JC, Black TA, Katz SI, Fan R, Yee SS, et al. Clinical Implications of Plasma-Based Genotyping With the Delivery of Personalized Therapy in Metastatic Non-Small Cell Lung Cancer. JAMA Oncol 2018 doi 10.1001/jamaoncol.2018.4305.

8. Sacher AG, Paweletz C, Dahlberg SE, Alden RS, O'Connell A, Feeney N, et al. Prospective Validation of Rapid Plasma Genotyping for the Detection of EGFR and KRAS Mutations in Advanced Lung Cancer. JAMA Oncol 2016;2(8):1014-22 doi 10.1001/jamaoncol.2016.0173. [PubMed: 27055085]

9. Lin KK, Harrell MI, Oza AM, Oaknin A, Ray-Coquard I, Tinker AV, et al. BRCA Reversion Mutations in Circulating Tumor DNA Predict Primary and Acquired Resistance to the PARP Inhibitor Rucaparib in High-Grade Ovarian Carcinoma. Cancer Discov 2018 doi 10.1158/2159-8290.Cd-18-0715.

10. Choudhury AD, Werner L, Francini E, Wei XX, Ha G, Freeman SS, et al. Tumor fraction in cellfree DNA as a biomarker in prostate cancer. JCI Insight 2018;3(21) doi 10.1172/jci.insight. 122109.

11. Wei T, Zhang Q, Li X, Su W, Li G, Ma T, et al. Monitoring Tumor Burden in Response to FOLFIRINOX Chemotherapy Via Profiling Circulating Cell-Free DNA in Pancreatic Cancer. Mol Cancer Ther 2019;18(1):196-203 doi 10.1158/1535-7163.Mct-17-1298. [PubMed: 30301865]

12. Valpione S, Gremel G, Mundra P, Middlehurst P, Galvani E, Girotti MR, et al. Plasma total cellfree DNA (cfDNA) is a surrogate biomarker for tumour burden and a prognostic biomarker for survival in metastatic melanoma patients. Eur J Cancer 2018;88:1-9 doi 10.1016/j.ejca. 2017.10.029. [PubMed: 29175734]

13. Gangadhar TC, Savitch SL, Yee SS, Xu W, Huang AC, Harmon S, et al. Feasibility of monitoring advanced melanoma patients using cell-free DNA from plasma. Pigment Cell Melanoma Res 2018;31(1):73-81 doi 10.1111/pcmr.12623. [PubMed: 28786531]

14. Stover DG, Parsons HA, Ha G, Freeman SS, Barry WT, Guo H, et al. Association of Cell-Free DNA Tumor Fraction and Somatic Copy Number Alterations With Survival in Metastatic TripleNegative Breast Cancer. J Clin Oncol 2018;36(6):543-53 doi 10.1200/jco.2017.76.0033. [PubMed: 29298117]

15. Iijima Y, Hirotsu Y, Amemiya K, Ooka Y, Mochizuki H, Oyama T, et al. Very early response of circulating tumour-derived DNA in plasma predicts efficacy of nivolumab treatment in patients with non-small cell lung cancer. Eur J Cancer 2017;86:349-57 doi 10.1016/j.ejca.2017.09.004. [PubMed: 29078173]

16. Pietrasz D, Pecuchet N, Garlan F, Didelot A, Dubreuil O, Doat S, et al. Plasma Circulating Tumor DNA in Pancreatic Cancer Patients Is a Prognostic Marker. Clinical cancer research : an official journal of the American Association for Cancer Research 2017;23(1):116-23 doi 10.1158/1078-0432.Ccr-16-0806. [PubMed: 27993964]

17. Heitzer E, Haque IS, Roberts CES, Speicher MR. Current and future perspectives of liquid biopsies in genomics-driven oncology. Nat Rev Genet 2019;20(2):71-88 doi 10.1038/s41576-018-0071-5. [PubMed: 30410101]

18. Thakkar JP, Dolecek TA, Horbinski C, Ostrom QT, Lightner DD, Barnholtz-Sloan JS, et al. Epidemiologic and molecular prognostic review of glioblastoma. Cancer Epidemiol Biomarkers Prev 2014;23(10):1985-96 doi 10.1158/1055-9965.Epi-14-0275. [PubMed: 25053711]

19. Patel AP, Tirosh I, Trombetta JJ, Shalek AK, Gillespie SM, Wakimoto H, et al. Single-cell RNAseq highlights intratumoral heterogeneity in primary glioblastoma. Science 2014;344(6190):1396401 doi 10.1126/science.1254257. [PubMed: 24925914]

20. Bettegowda C, Sausen M, Leary RJ, Kinde I, Wang Y, Agrawal N, et al. Detection of circulating tumor DNA in early- and late-stage human malignancies. Science translational medicine 2014;6(224):224ra24 doi 10.1126/scitranslmed.3007094. 
21. Volik S, Alcaide M, Morin RD, Collins C. Cell-free DNA (cfDNA): Clinical Significance and Utility in Cancer Shaped By Emerging Technologies. Mol Cancer Res 2016;14(10):898-908 doi 10.1158/1541-7786.Mcr-16-0044. [PubMed: 27422709]

22. Boisselier B, Gallego Perez-Larraya J, Rossetto M, Labussiere M, Ciccarino P, Marie Y, et al. Detection of IDH1 mutation in the plasma of patients with glioma. Neurology 2012;79(16):1693-8 doi 10.1212/WNL.0b013e31826e9b0a. [PubMed: 23035067]

23. Panditharatna E, Kilburn LB, Aboian MS, Kambhampati M, Gordish-Dressman H, Magge SN, et al. Clinically Relevant and Minimally Invasive Tumor Surveillance of Pediatric Diffuse Midline Gliomas Using Patient-Derived Liquid Biopsy. Clinical cancer research : an official journal of the American Association for Cancer Research 2018;24(23):5850-9 doi 10.1158/1078-0432.Ccr-18-1345. [PubMed: 30322880]

24. Schwaederle M, Husain H, Fanta PT, Piccioni DE, Kesari S, Schwab RB, et al. Detection rate of actionable mutations in diverse cancers using a biopsy-free (blood) circulating tumor cell DNA assay. Oncotarget 2016;7(9):9707-17 doi 10.18632/oncotarget.7110. [PubMed: 26848768]

25. Piccioni DE, Achrol AS, Kiedrowski LA, Banks KC, Boucher N, Barkhoudarian G, et al. Analysis of cell-free circulating tumor DNA in 419 patients with glioblastoma and other primary brain tumors. CNS Oncol 2019 doi 10.2217/cns-2018-0015.

26. Ellingson BM, Chung C, Pope WB, Boxerman JL, Kaufmann TJ. Pseudoprogression, radionecrosis, inflammation or true tumor progression? challenges associated with glioblastoma response assessment in an evolving therapeutic landscape. J Neurooncol 2017;134(3):495-504 doi 10.1007/s11060-017-2375-2. [PubMed: 28382534]

27. Stupp R, Mason WP, van den Bent MJ, Weller M, Fisher B, Taphoorn MJ, et al. Radiotherapy plus concomitant and adjuvant temozolomide for glioblastoma. N Engl J Med 2005;352(10):987-96 doi 10.1056/NEJMoa043330. [PubMed: 15758009]

28. Fawzy A, Sweify KM, El-Fayoumy HM, Nofal N. Quantitative analysis of plasma cell-free DNA and its DNA integrity in patients with metastatic prostate cancer using ALU sequence. J Egypt Natl Canc Inst 2016;28(4):235-42 doi 10.1016/j.jnci.2016.08.003. [PubMed: 27634416]

29. Lanman RB, Mortimer SA, Zill OA, Sebisanovic D, Lopez R, Blau S, et al. Analytical and Clinical Validation of a Digital Sequencing Panel for Quantitative, Highly Accurate Evaluation of Cell-Free Circulating Tumor DNA. PLoS One 2015;10(10):e0140712 doi 10.1371/journal.pone.0140712. [PubMed: 26474073]

30. Zill OA, Banks KC, Fairclough SR, Mortimer SA, Vowles JV, Mokhtari R, et al. The Landscape of Actionable Genomic Alterations in Cell-Free Circulating Tumor DNA from 21,807 Advanced Cancer Patients. Clinical cancer research : an official journal of the American Association for Cancer Research 2018;24(15):3528-38 doi 10.1158/1078-0432.Ccr-17-3837. [PubMed: 29776953]

31. Kickingereder P, Isensee F, Tursunova I, Petersen J, Neuberger U, Bonekamp D, et al. Automated quantitative tumour response assessment of MRI in neuro-oncology with artificial neural networks: a multicentre, retrospective study. Lancet Oncol 2019;20(5):728-40 doi 10.1016/ s1470-2045(19)30098-1. [PubMed: 30952559]

32. Yushkevich PA, Piven J, Hazlett HC, Smith RG, Ho S, Gee JC, et al. User-guided 3D active contour segmentation of anatomical structures: significantly improved efficiency and reliability. Neuroimage 2006;31(3):1116-28 doi 10.1016/j.neuroimage.2006.01.015. [PubMed: 16545965]

33. Barnes SR, Ng TS, Santa-Maria N, Montagne A, Zlokovic BV, Jacobs RE. ROCKETSHIP: a flexible and modular software tool for the planning, processing and analysis of dynamic MRI studies. BMC medical imaging 2015;15:19 doi 10.1186/s12880-015-0062-3. [PubMed: 26076957]

34. Lapin M, Oltedal S, Tjensvoll K, Buhl T, Smaaland R, Garresori H, et al. Fragment size and level of cell-free DNA provide prognostic information in patients with advanced pancreatic cancer. $\mathrm{J}$ Transl Med 2018;16(1):300 doi 10.1186/s12967-018-1677-2. [PubMed: 30400802]

35. Garlan F, Laurent-Puig P, Sefrioui D, Siauve N, Didelot A, Sarafan-Vasseur N, et al. Early Evaluation of Circulating Tumor DNA as Marker of Therapeutic Efficacy in Metastatic Colorectal Cancer Patients (PLACOL Study). Clinical cancer research : an official journal of the American Association for Cancer Research 2017;23(18):5416-25 doi 10.1158/1078-0432.Ccr-16-3155. [PubMed: 28576867] 
36. Wang W, Kong P, Ma G, Li L, Zhu J, Xia T, et al. Characterization of the release and biological significance of cell-free DNA from breast cancer cell lines. Oncotarget 2017;8(26):43180-91 doi 10.18632/oncotarget.17858. [PubMed: 28574818]

37. Jahr S, Hentze H, Englisch S, Hardt D, Fackelmayer FO, Hesch RD, et al. DNA fragments in the blood plasma of cancer patients: quantitations and evidence for their origin from apoptotic and necrotic cells. Cancer Res 2001;61(4):1659-65. [PubMed: 11245480]

38. Mair R, Mouliere F, Smith CG, Chandrananda D, Gale D, Marass F, et al. Measurement of Plasma Cell-Free Mitochondrial Tumor DNA Improves Detection of Glioblastoma in Patient-Derived Orthotopic Xenograft Models. Cancer Res 2019;79(1):220-30 doi 10.1158/0008-5472.Can-18-0074. [PubMed: 30389699]

39. Brennan CW, Verhaak RG, McKenna A, Campos B, Noushmehr H, Salama SR, et al. The somatic genomic landscape of glioblastoma. Cell 2013;155(2):462-77 doi 10.1016/j.cell.2013.09.034. [PubMed: 24120142]

40. Forshew T, Murtaza M, Parkinson C, Gale D, Tsui DW, Kaper F, et al. Noninvasive identification and monitoring of cancer mutations by targeted deep sequencing of plasma DNA. Science translational medicine 2012;4(136):136ra68 doi 10.1126/scitranslmed.3003726.

41. Aldape K, Brindle KM, Chesler L, Chopra R, Gajjar A, Gilbert MR, et al. Challenges to curing primary brain tumours. Nature Reviews Clinical Oncology 2019 doi 10.1038/s41571-019-0177-5.

42. Hu Y, Ulrich BC, Supplee J, Kuang Y, Lizotte PH, Feeney NB, et al. False-Positive Plasma Genotyping Due to Clonal Hematopoiesis. Clinical cancer research : an official journal of the American Association for Cancer Research 2018;24(18):4437-43 doi 10.1158/1078-0432.Ccr-18-0143. [PubMed: 29567812]

43. Stetson D, Ahmed A, Xu X, Nuttall BRB, Lubinski TJ, Johnson JH, et al. Orthogonal Comparison of Four Plasma NGS Tests With Tumor Suggests Technical Factors are a Major Source of Assay Discordance. JCO Precision Oncology 2019(3):1-9 doi 10.1200/po.18.00191.

44. Martinez-Ricarte F, Mayor R, Martinez-Saez E, Rubio-Perez C, Pineda E, Cordero E, et al. Molecular Diagnosis of Diffuse Gliomas through Sequencing of Cell-Free Circulating Tumor DNA from Cerebrospinal Fluid. Clinical cancer research : an official journal of the American Association for Cancer Research 2018;24(12):2812-9 doi 10.1158/1078-0432.Ccr-17-3800. [PubMed: 29615461]

45. De Mattos-Arruda L, Mayor R, Ng CK, Weigelt B, Martinez-Ricarte F, Torrejon D, et al. Cerebrospinal fluid-derived circulating tumour DNA better represents the genomic alterations of brain tumours than plasma. Nat Commun 2015;6:8839 doi 10.1038/ncomms9839. [PubMed: 26554728]

46. Miller AM, Shah RH, Pentsova EI, Pourmaleki M, Briggs S, Distefano N, et al. Tracking tumour evolution in glioma through liquid biopsies of cerebrospinal fluid. Nature 2019;565(7741):654-8 doi 10.1038/s41586-019-0882-3. [PubMed: 30675060]

47. Underhill HR, Kitzman JO, Hellwig S, Welker NC, Daza R, Baker DN, et al. Fragment Length of Circulating Tumor DNA. PLoS Genet 2016;12(7):e1006162 doi 10.1371/journal.pgen.1006162. [PubMed: 27428049]

48. Zhu L, Cheng G, Ye D, Nazeri A, Yue Y, Liu W, et al. Focused Ultrasound-enabled Brain Tumor Liquid Biopsy. Sci Rep 2018;8(1):6553 doi 10.1038/s41598-018-24516-7. [PubMed: 29700310]

49. Ellingson BM, Abrey LE, Garcia J, Chinot O, Wick W, Saran F, et al. Post-chemoradiation volumetric response predicts survival in newly diagnosed glioblastoma treated with radiation, temozolomide, and bevacizumab or placebo. Neuro Oncol 2018;20(11):1525-35 doi 10.1093/ neuonc/noy064. [PubMed: 29897562]

50. Prakash K, Aggarwal S, Bhardwaj S, Ramakrishna G, Pandey CK. Serial perioperative cell-free DNA levels in donors and recipients undergoing living donor liver transplantation. Acta Anaesthesiol Scand 2017;61(9):1084-94 doi 10.1111/aas.12947. [PubMed: 28766696] 


\section{Translational Relevance}

Glioblastoma (GBM) is the most common primary malignant brain tumor in adults and carries a dismal prognosis. Significant challenges in the care of patients with GBM include marked molecular heterogeneity, inability of current imaging methods to accurately monitor tumor progression, and difficulty in refining prognosis for individual patients treated with standard chemoradiotherapy. Each of these challenges is further compounded by the invasive nature of brain surgery, making repeated tumor biopsies or resections impractical. The results of this study support the possibility of using plasma cell-free DNA (cfDNA) as a prognostic tool, a surrogate of tumor burden and progression, and a means of obtaining additional molecular profiling in patients with GBM. If our results are confirmed in larger studies, the care of patients with GBM could be significantly improved through reduction in morbid procedures, enhanced assessment of response to therapy, and increased use of next-generation sequencing (NGS) to inform personalized therapy. 


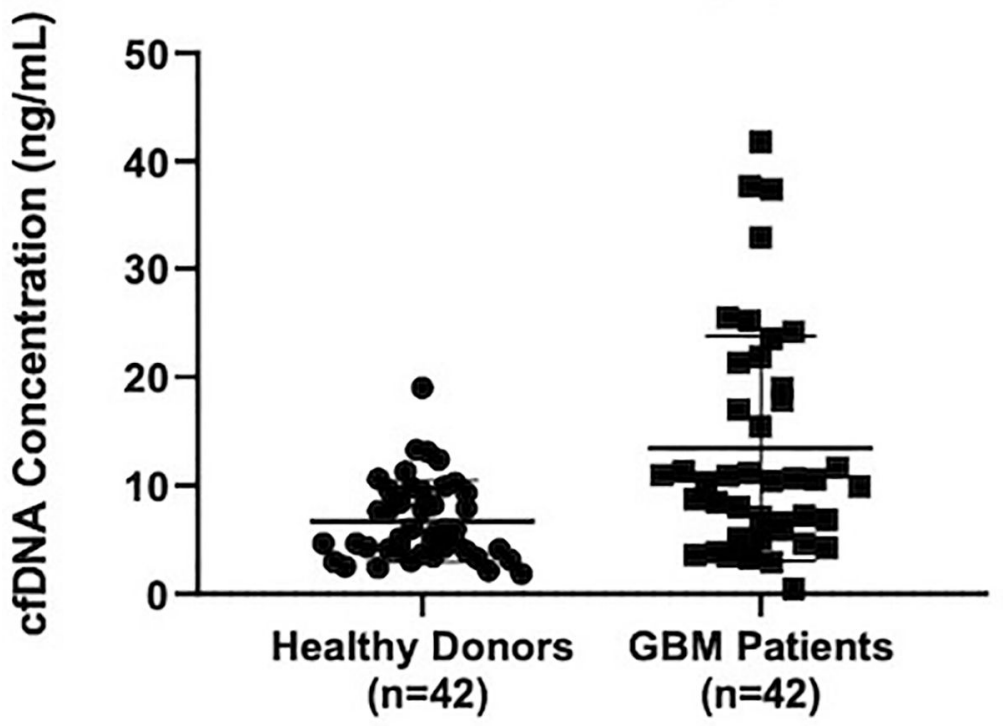

Figure 1.

Plasma cell-free DNA (cfDNA) concentration in age-matched healthy donors (known cancers and/or inflammatory diseases excluded) versus patients with newly diagnosed GBM prior to surgical resection. 


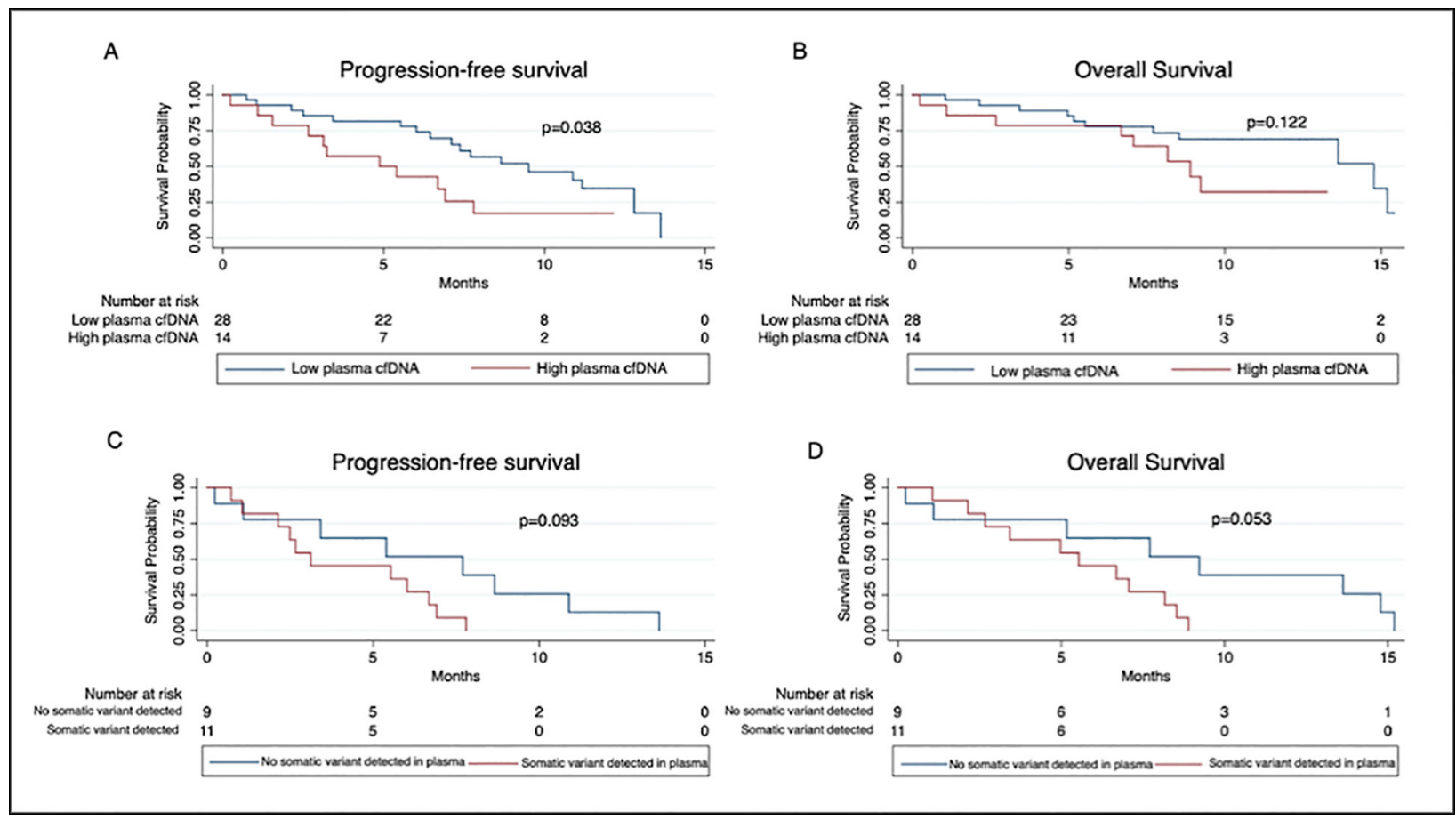

Figure 2.

Kaplan-Meier curves for progression-free and overall survival (months from initial surgery) of glioblastoma patients according to $(\mathbf{A})$ and $(\mathbf{B})$ baseline plasma cell-free DNA (cfDNA) concentration, and (C) and (D) detectability of $\geq 1$ versus no somatic mutations in cfDNA. 


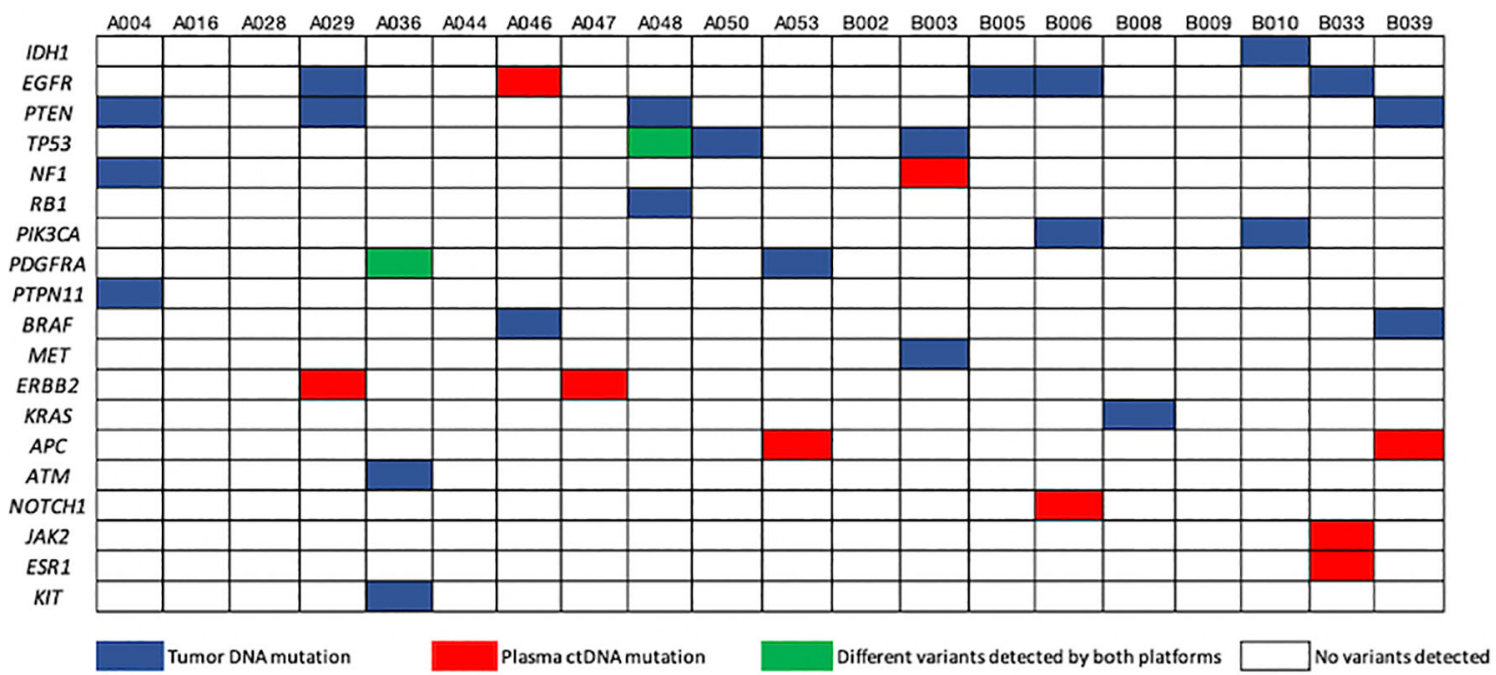

Figure 3.

Mutational analysis for 20 patients for whom both plasma and tissue samples were obtained concurrently and sequenced. A detailed summary of all disease-associated somatic variants covered and called by both the tissue tumor DNA and circulating tumor DNA (ctDNA) platforms are included. 


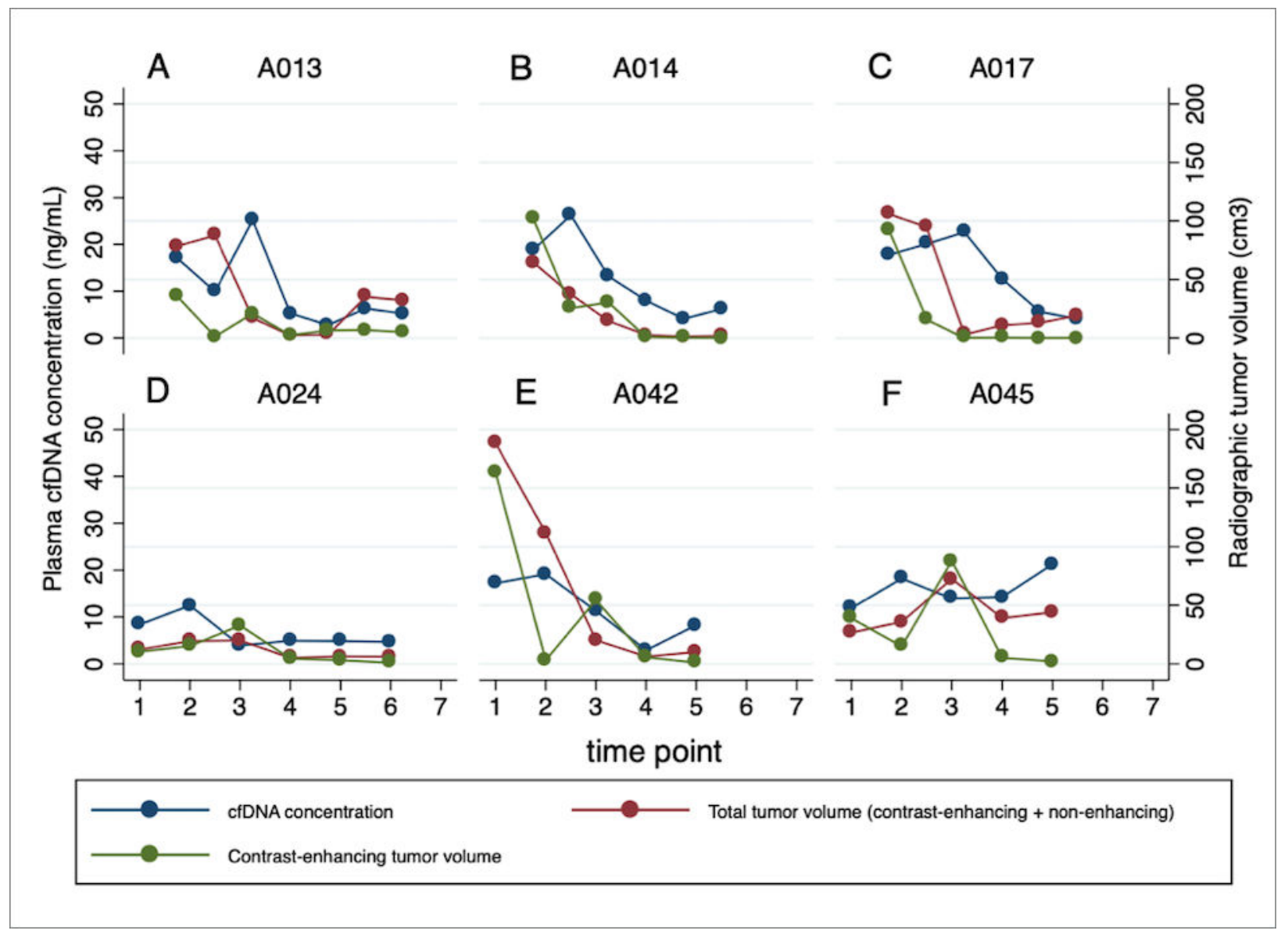

Figure 4.

cfDNA dynamics and radiographic tumor burden for subjects enrolled on the longitudinal portion of the study who had not yet experienced tumor progression by the time of the data cutoff for this analysis. Time points are the following: 1 (pre-operative), 2 (post-operative), 3 (radiation simulation), 4 (1-month post-radiation scan), 5 ( $1^{\text {st }}$ follow up scan), 6 ( $2^{\text {nd }}$ follow up scan), and 7 ( $3^{\text {rd }}$ follow up scan). All subjects had stable disease and were continued on maintenance temozolomide at all time points. cfDNA generally remained low and stable in these subjects, although there were minor increases (all less than $5 \mathrm{ng} / \mathrm{mL}$ changes) at the most recent time point in subjects A014 (B), A042 (E), and A045 (F). 

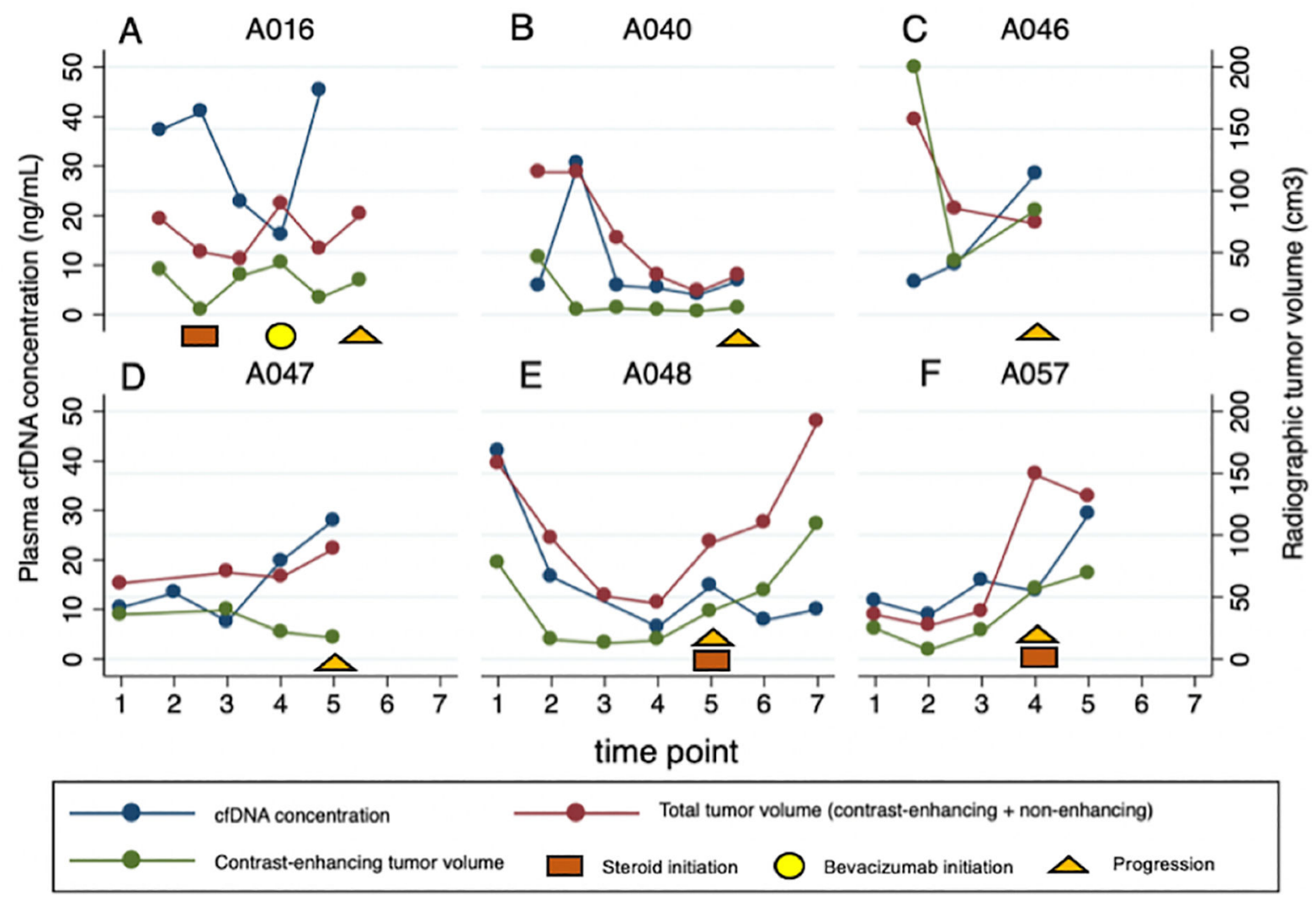

Figure 5.

cfDNA dynamics and radiographic tumor burden for subjects enrolled on the longitudinal portion of the study who had confirmed progressive disease prior to the data cutoff for this analysis. Time points are the following: 1 (pre-operative), 2 (post-operative), 3 (radiation simulation), 4 (1-month post-radiation scan), 5 ( $1^{\text {st }}$ follow up scan), 6 ( $2^{\text {nd }}$ follow up scan), and 7 ( $3^{\text {rd }}$ follow up scan). A, Subject A016 had increased tumor volume on the first postradiation MRI, concerning for progressive tumor versus pseudoprogression. The subject was steroid-dependent, and bevacizumab was initiated with radiographic response (time point \#5). However, cfDNA rose rapidly during this time, which was followed over the subsequent month (time point \#6) by radiographic tumor progression, clinical decline, and death of the subject from disease progression. B, Subject A040 developed tumor progression after 3 cycles of temozolomide, accompanied by a modest rise in cfDNA. C, Subject A046 experienced early disease progression on the first post-radiation MRI, accompanied by a dramatic rise in cfDNA. The subject died from disease progression 2 months later. D, Subject A047 had a significant rise in cfDNA between the radiation planning and first postradiation MRIs. Although this rise was initially accompanied by a decline in radiographic tumor volume, cfDNA concentration continued to increase and was followed by nonenhancing tumor progression and clinical decline, resulting in subject's death from disease progression within 4 weeks. E, Subject A048 had radiographic increase in tumor volume 3 months after completion of radiation (time point \#5). Steroids were initiated and an additional cycle of temozolomide was administered. One month later (time point \# 6), there 
was only minimal continued increase in tumor size with no clinical decline, and cfDNA concentration decreased. Ongoing pseudoprogression was clinically suspected, and the subject completed another cycle of temozolomide. At the next time point (\# 7), the tumor had grown considerably, accompanied by resumed increase in cfDNA. The subject underwent repeat craniotomy with histopathology demonstrating recurrent GBM and died 1 month later. F, Subject A057 had increased tumor volume on the first post-radiation MRI. A repeat MRI one month later showed continued increase in contrast-enhancing tumor, accompanied by dramatic rise in cfDNA. The subject underwent repeat craniotomy with histopathology confirming recurrent GBM. 
Table 1.

Baseline characteristics of adult glioblastoma cohort by pre-operative plasma cfDNA concentration

\begin{tabular}{|c|c|c|c|c|}
\hline Characteristic & All subjects $(\mathrm{N}=\mathbf{4 2})$ & $\begin{array}{l}\text { Low plasma cfDNA } \\
(\mathrm{N}=\mathbf{2 8})\end{array}$ & $\begin{array}{l}\text { High plasma cfDNA } \\
(\mathrm{N}=14)\end{array}$ & p-value \\
\hline Median age, years (range) & $65(20-81)$ & $65(20-77)$ & $69(41-81)$ & 0.20 \\
\hline \multicolumn{5}{|l|}{ Sex - no. $(\%)$} \\
\hline Male & $22(52)$ & $13(46)$ & $9(64)$ & 0.28 \\
\hline Female & $20(48)$ & $15(54)$ & $5(36)$ & \\
\hline \multicolumn{5}{|l|}{ Median tumor volume, $\mathrm{cm}^{3}$ (range) } \\
\hline Contrast-enhancing ( $\mathrm{T} 1$ post-contrast) & $16.9(2.1-79.5)$ & $16.6(2.1-79.5)$ & $16.9(7.1-36.7)$ & 0.97 \\
\hline Non-enhancing (T2/FLAIR) & $72.9(10.2-434.5)$ & $55.9(10.2-434.5)$ & $100.0(16.4-285.4)$ & 0.14 \\
\hline Total (contrast-enhancing + T2/FLAIR) & $81.5(12.2-464.2)$ & $76.7(12.2-464.2)$ & $128.1(26.5-304.0)$ & 0.16 \\
\hline \multicolumn{5}{|l|}{ Surgical resection - no. (\%) } \\
\hline Gross Total & $16(38)$ & $12(43)$ & $4(29)$ & 0.54 \\
\hline Near Total & $16(38)$ & $11(39)$ & $5(36)$ & \\
\hline Partial & $7(17)$ & $4(14)$ & $3(21)$ & \\
\hline Biopsy Only & $3(7)$ & $1(4)$ & $2(14)$ & \\
\hline \multicolumn{5}{|l|}{ Karnofsky performance status - no. (\%) } \\
\hline$\leq 60$ & $7(17)$ & $5(18)$ & $2(14)$ & 1.0 \\
\hline $70-80$ & $15(36)$ & $10(36)$ & $5(36)$ & \\
\hline $90-100$ & $20(47)$ & $13(46)$ & $7(50)$ & \\
\hline \multicolumn{5}{|l|}{$I D H 1 / 2^{*}$ mutation status - no. $(\%)$} \\
\hline Mutant & $7(17)$ & $4(14)$ & $3(21)$ & 0.43 \\
\hline Wild-type & $35(83)$ & $24(86)$ & $11(79)$ & \\
\hline \multicolumn{5}{|l|}{$M G M T^{* * *}$ promoter methylation status - no. (\%) } \\
\hline Methylated & $26(62)$ & $19(68)$ & $7(50)$ & 0.26 \\
\hline Unmethylated & $16(38)$ & $9(32)$ & $7(50)$ & \\
\hline \multicolumn{5}{|l|}{ Tumor somatic variants by tissue NGS ${ }^{* * *}$ - no. $(\%)$} \\
\hline TP53 mutation & $10(24)$ & $7(25)$ & $3(21)$ & 1.0 \\
\hline PTEN mutation & $7(17)$ & $3(11)$ & $4(29)$ & 0.20 \\
\hline NF1 mutation & $5(12)$ & $2(7)$ & $3(21)$ & 0.25 \\
\hline $\begin{array}{l}E G F R \text { (point mutation, EGFRvIII, or copy number } \\
\text { gain) }\end{array}$ & $9(21)$ & $5(18)$ & $4(29)$ & 0.45 \\
\hline STAG2 mutation & $4(10)$ & $2(7)$ & $2(14)$ & 0.59 \\
\hline PTPN11 mutation & $3(7)$ & $1(4)$ & $2(14)$ & 0.25 \\
\hline PDGFRA (point mutation or copy number gain) & $4(10)$ & $4(14)$ & $0(0)$ & 0.28 \\
\hline$P I K 3 C A$ & $3(7)$ & $2(7)$ & $1(7)$ & 1.0 \\
\hline
\end{tabular}

* IDH; Isocitrate dehydrogenase

*** MGMT; O6-methylguanine-methyltransferase

*** NGS; Next-generation sequencing; only most common disease-associated variants in the cohort are listed

Clin Cancer Res. Author manuscript; available in PMC 2020 July 15. 